\title{
Adropin and irisin in arterial hypertension, diabetes mellitus and chronic kidney disease
}

\author{
Magdalena Maciorkowska ${ }^{1, A-D}$, Dominika Musiałowska ${ }^{1, B, C}$, Jolanta Małyszko ${ }^{2, E, F}$ \\ 1 Second Department of Nephrology and Hypertension with Dialysis Unit, Medical University of Bialystok, Poland \\ ${ }^{2}$ Department of Nephrology, Dialysis and Internal Medicine, Medical University of Warsaw, Poland \\ A - research concept and design; $B$ - collection and/or assembly of data; $C$ - data analysis and interpretation; \\ $D$ - writing the article; $E$ - critical revision of the article; $F$ - final approval of the article
}

Address for correspondence

Magdalena Maciorkowska

E-mail: magdalena.maciorkowska@gmail.com

Funding sources

None declared

Conflict of interest

None declared

Received on January 25, 2018

Reviewed on June 17, 2018

Accepted on February 18, 2019

Published online on November 18, 2019

\begin{abstract}
Despite great advances in medicine, the proper treatment of arterial hypertension (AH), diabetes mellitus (DM) and chronic kidney disease (CKD) remains a major challenge. Untreated, undiagnosed AH or DM may lead to the development of CKD and consequently to the occurrence of cardiovascular events. Adropin and irisin are newly discovered proteins which may play a role in the development and progression of the chronic diseases mentioned above. Endothelium dysfunction could be a bonding point. The following review paper focuses on adropin and risisin concentrations and their correlations in AH, DM and CKD. Lower adropin concentrations have been measured in patients with primary AH when compared to healthy volunteers. Irisin has reduced blood pressure on nitric oxide (NO)-dependent pathways in experimental studies; a negative correlation between irisin and blood pressure values has also been observed in preeclamptic women. Irisin also plays a role in insulin sensitivity and metabolic disorders. Lower irisin levels have been observed in patients with DM type 2 in comparison to a nondiabetic control group. It is also lower in the serum of pregnant women with gestational DM. A negative correlation between irisin and estimated glomerular filtration rate (EGFR) has also been noted. Adropin and irisin are newly described myokines measured in human plasma in healthy and disease status. Their exact function has not been specified yet and requires further studies.
\end{abstract}

Key words: diabetes mellitus, hypertension, kidney, proteins
Cite as

Maciorkowska M, Musiałowska D, Małyszko J. Adropin and irisin in arterial hypertension, diabetes mellitus and chronic kidney disease. Adv Clin Exp Med. 2019;28(11):1571-1575. doi:10.17219/acem/104551

DOI

10.17219/acem/104551

\section{Copyright}

Copyright by Author(s)

This is an article distributed under the terms of the

Creative Commons Attribution Non-Commercial License

(http://creativecommons.org/licenses/by-nc-nd/4.0/) 
The relationship between arterial hypertension $(\mathrm{AH})$, diabetes mellitus (DM) and cardiovascular as well as renal events has been demonstrated in many available studies and meta-analyses. ${ }^{1-3}$ Despite huge progress in medicine, the number of patients with $\mathrm{AH}$ is still rising and a large percentage of people remain undiagnosed for a long time. ${ }^{4,5}$ These facts demand further research to find the pathophysiological pathways of these disorders in order to develop new therapeutic possibilities. Adropin and irisin, newly characterized proteins, may be important elements in the pathophysiological pathways of these disorders. They are both myokines regulating energy homeostasis and metabolic processes. ${ }^{6,7}$

Adropin was characterized for the first time in 2008 as a secreted peptide consisting of 76 amino acids. This newly discovered peptide hormone is encoded by the ENHO gene and is expressed in the liver, kidney, heart, small intestine, body fluids, and central nervous system. Researchers suggest that adropin has a role in endothelial dysfunction, insulin resistance and energy homeostasis. ${ }^{8}$ Recent data confirms that this bioactive protein has an ability to take part in cell-to-cell communication. ${ }^{9}$

Irisin, a membrane protein whose precursor is the fibronectin type III domain containing protein 5 (FNDC5), has been described as a hormone produced by myocytes. Experimental studies have shown that its level is maintained by PPAR- $\gamma$ coactivator -1 (PGC1)- $\alpha$ in the circulation. The irisin sequence has been measured with mass spectrometry and a great resemblance has been found between humans and mice..$^{10,11}$

In this review, we will focus on the possible role of irisin and adropin in patients with chronic kidney disease (CKD), $\mathrm{DM}$ and $\mathrm{AH}$.

\section{Adropin, irisin and hypertension}

Hypertension is a risk factor for cardiovascular events. Many patients with diagnosed $\mathrm{AH}$ have inadequate blood pressure control. Insufficient or inadequate treatment can contribute to the end-stage CKD. ${ }^{12}$ Available population studies suggest that a large percentage of the population suffer from high blood pressure. ${ }^{13}$ Published experimental studies indicate an association between serum adropin levels and $\mathrm{AH}$. Researchers have analyzed adropin levels in a group of 40 obese hypertensive children and 15 healthy volunteers; no correlation was found between adropin concentration and high blood pressure, but interestingly, a significantly lower concentration of adropin was measured in the obese children. ${ }^{14}$ A nother study used adropin and endothelin-1 (ET-1) enzyme-linked immunosorbent assay (ELISA) kits to show an association between adropin and endothelin-1 concentrations in patients with essential AH. A significantly lower adropin level was observed in participants with primary $\mathrm{AH}$ when compared to the control group. The authors concluded that adropin may be a predictor of primary AH. The study also showed that the molecular weight of adropin is very low and that this hormone is easily filtered by the kidneys. Consequently, the serum level of adropin is lower than its concentration in urine, especially in hypertensive patients. ${ }^{15}$

Adult patients with blood pressure values over 180/110 mm Hg had significantly lower serum levels of adropin than the normotensive controls. However, adropin levels cannot be used as a marker of target organ damage (TOD) because of its similar levels in the groups with and without TOD. ${ }^{16}$

There are also studies confirming an association between irisin and blood pressure. Irisin mediates energy homeostasis and it is produced by muscle cells, among others. On the basis of these facts and the correlation between metabolic disorders and $\mathrm{AH}$, the association between low serum irisin levels and arterial relaxation in hypertensive and normotensive rats was analyzed. Irisin stimulates the production of nitric oxide (NO) and endothelial NO synthase (eNOS). Moreover, the vasorelaxing effect was connected with activation of the 5-AMP-activated protein kinase (AMPK) and blocking of protein kinase B (Akt). Summarizing, the researchers observed that irisin reduced blood pressure on the AMPK-Akt-eNOS-NO dependent pathway and led to vasorelaxation in hypertensive rats. This mechanism was dose-dependent. A higher level of NO improved endothelial function. ${ }^{17}$

Another study focused on correlations between irisin and $\mathrm{AH}$ in pregnant and preeclamptic women. The researchers used ELISA kits to analyze the level of irisin in the blood. The study included 67 pregnant patients (31 healthy volunteers and 36 preeclamptic women). Interestingly, a negative correlation between irisin and systolic and diastolic blood pressure was observed in preeclamptic women. A negative correlation between age and irisin levels in preeclamptic women was also noticed. ${ }^{18}$ Two years before, the influence of irisin on blood pressure was analyzed in an experimental study by the same researchers. They showed that central administration of this peptide hormone raised blood pressure, while its peripheral administration caused a hypotensive effect in both hypertensive and healthy rats. ${ }^{19}$ The exact mechanisms regarding the different effects of irisin on blood pressure are unknown. Weizhen et al. suggested that central irisin administration raises blood pressure as a consequence of increased cardiac output by activation of the hypothalamus. The hypothalamus influences blood pressure through adrenergic sympathetic activity. Irisin regulates blood pressure peripherally by influencing blood vessels, specifically in endothelial and smooth muscle cells. It could be a messenger connecting the brain and the cardiovascular system. ${ }^{20}$

\section{Adropin, irisin and diabetes mellitus}

Diabetes mellitus type 2 is a metabolic disorder characterized by increased blood glucose, caused by a lack of insulin and/or insulin resistance. Diabetes mellitus 
is a globally widespread health problem. Long-term hyperglycemia causes complications such as coronary artery disease, diabetic retinopathy and impaired renal function. ${ }^{21}$ There are many experimental and clinical studies concerning irisin concentrations in subjects with DM type 2. It has been proven that irisin plays a role in insulin sensitivity and metabolic disorders. ${ }^{11}$ Lower irisin levels have been observed in patients with DM type 2 in comparison to a nondiabetic control group. ${ }^{22}$ On the other hand, obese nondiabetic patients had significantly higher levels of irisin in comparison with thin nondiabetic participants. Irisin was closely related to body mass index (BMI), homeostatic model assessment of insulin resistance (HOMA-IR) and fasting insulin. ${ }^{23}$ Chinese researchers found that serum irisin was significantly lower in 104 patients with newly diagnosed DM type 2 as opposed to the control subjects (104 healthy volunteers). ${ }^{22}$ A meta-analysis carried out by Zhang et al. demonstrated significantly lower irisin levels in patients with newly diagnosed DM. ${ }^{24}$

The same results were also observed in the maternal serum levels of irisin in women with gestational DM. ${ }^{25}$ Irisin concentrations were significantly lower in the study group in comparison to healthy pregnant women. Interestingly, there was no statistically significant correlation between circulating irisin levels in cord blood in the same study and the control group of pregnant women. ${ }^{25}$

In addition, decreasing adropin expression was observed in cord serum and also in maternal blood in women with gestational DM. ${ }^{26,27}$ Increased levels of adropin seemed to influence abnormal fetal growth in women with gestational DM, possibly through placentation dysfunctions. No significant differences in adropin levels in pregnant women with gestational DM type 1 or gestational DM type 2 were noticed. Moreover, a positive correlation between adropin and glycated hemoglobin (HbA1c) was observed. ${ }^{28}$

Not many studies have analyzed irisin and adropin concentrations in breast milk. Lower concentrations of these hormones have been observed in patients with gestational DM. Circulating irisin and adropin levels in breast milk reflected their concentrations in plasma. ${ }^{29}$

Another research group measured irisin levels in patients with DM type 2 and suggested that plasma irisin seems to be connected to metabolic factors in healthy subjects but not in diabetic patients. ${ }^{30}$ Serum levels of irisin in the control group were associated with total cholesterol, triglycerides, age, diastolic blood pressure, and fasting blood glucose. As in earlier studies, the authors observed decreased irisin concentrations in patients with DM type $2 .^{30}$ The data presented reflected a reduced level of irisin in patients with renal insufficiency and DM type 2. Blood pressure and age have been shown to correlate with irisin in patients with DM type 2 and normal renal function. ${ }^{31}$ A research team from China found that irisin ameliorated disturbed endothelial function in patients with DM type $2 .^{32}$ They observed improvements in vascular function after the administration of $0.5 \mathrm{mg} / \mathrm{kg} /$ day of irisin in mice suffering from DM type 2 over a period of 2 weeks. The authors also showed protective effects of irisin on the diabetic aortic endothelium. Some aorta segments were exposed to irisin $(1 \mu \mathrm{g} / \mathrm{mL})$ ex vivo. In their observations, irisin reduced the production of NO synthase, the glycosylated subunit component of NADPH oxidase (gp91phox) as well as peroxynitrite. Irisin may have an inhibitory effect on PKC-beta/NADPH oxidase and NF-kB/iNOS pathways. ${ }^{32}$ Lower irisin levels were measured in patients with newly diagnosed DM type 2 . Interestingly, multivariate regression showed a positive association between irisin concentrations and flow-mediated dilation levels. ${ }^{33}$ The same authors, in an experimental study, showed that excessive expression of FNDC5/irisin ameliorated insulin sensitivity and decreased hyperglycemia as well as hyperlipidemia. ${ }^{34}$

A study published in 2017 presented a correlation between irisin and the AMP-activated protein kinase (AMPK) pathway. Irisin improved glucose and lipid metabolism and lowered the insulin resistance of hepatic cells. ${ }^{35}$ A Korean research group carried out the first prospective study focused on serum irisin levels as a risk factor for the occurrence of incident DM. ${ }^{11}$ The study included 3,500 patients. Incident DM was diagnosed in patients with a level of fasting glucose $\geq 126 \mathrm{mg} / \mathrm{dL}$, or on the basis of glycated hemoglobin $\geq 6.5 \%$, or in patients using medicines to lower glucose levels during the study. Interestingly, the authors suggested that circulating irisin can be used as a factor to predict DM in a healthy population. ${ }^{11}$ In a meta-analysis carried out in 2016, irisin levels correlated positively with an insulin resistance index. ${ }^{36}$ In this meta-analysis, 17 studies were taken into consideration, involving a total of 1,912 non-diabetic, non-pregnant adults. The authors observed a stronger positive correlation between irisin levels and insulin resistance subgroups of this meta-analysis with fasting blood glucose $\geq 6.1 \mathrm{mmol} / \mathrm{L}$ in comparison to patients with fasting blood glucose $<6.1 \mathrm{mmol} / \mathrm{L} .{ }^{36}$ This correlation was statistically significant in Americans and Asians but not in Europeans. ${ }^{36-38}$

Adropin concentrations in the serum and various organs of rats with streptozotocin-induced DM were analyzed. ${ }^{39}$ The myokine levels were measured with ELISA kit and determined on the basis of the mass of the tissues. The concentration of adropin in liver, pancreas, kidney and cerebellum tissues as well as in serum was higher in rats with DM in comparison to the healthy rats. ${ }^{39}$ A group of Turkish researchers published results pointing to a correlation between adropin concentration and endothelium dysfunction based on flow-mediated dilatation (FDM) in volunteers with DM type 2. They observed lower serum adropin levels in individuals with endothelial dysfunction in comparison to a control group. It is important that serum adropin appeared as a marker of endothelial dysfunction. ${ }^{40}$ It has been suggested that adropin may be a potential predictor of coronary artery disease: adropin concentrations in diabetic patients correlated with 
the advancement of coronary atherosclerosis. ${ }^{41}$ It has not been confirmed if adropin has the same results in experimental and clinical studies, nor if experimental studies may be extrapolated to humans.

\section{Irisin, adropin and kidney function}

Chronic kidney disease, an increasingly widespread health problem, is diagnosed on the basis of a reduced estimated glomerular filtration rate (eGFR) as well as albuminuria. Knowledge and understanding of CKD predictors can prevent complications and protect against the development of the terminal stage of renal failure. One risk factor for CKD is obesity, which is currently considered a worldwide epidemic problem. ${ }^{42,43}$

In patients with CKD, irisin was associated with fat mass, BMI and eGFR. The lowest irisin levels were observed in patients with the $5^{\text {th }}$ stage of CKD. ${ }^{31}$

Adult obese Chinese patients with higher concentrations of irisin had considerably lower incidence of CKD in comparison to obese subjects with reduced irisin levels. ${ }^{44}$ A correlation between irisin and eGFR was also observed: The group of patients in the $5^{\text {th }}$ stage of CKD had the lowest level of this hormone. No link between circulating irisin and microalbuminuria was confirmed. ${ }^{30}$ Another study showed that serum irisin was decreased in the CKD group. Furthermore, they found that in peritoneal dialyzed patients serum irisin levels were higher than in hemodialyzed patients. On the one hand, the research group indicated that glomerular filtration rate (eGFR) and plasma bicarbonate were identified as irisin concentration predictors, but on the other hand, no association of this myokine and body composition markers was observed. ${ }^{45}$ Another study reported significantly lower plasma irisin concentrations in hemodialysis patients when compared to healthy volunteers; moreover, no association between irisin levels after resistance exercise training (RETP) in hemodialysis individuals was observed. ${ }^{46}$

Another study investigated whether irisin levels may be taken as a risk factor of sarcopenia and carotid atherosclerosis in peritoneal dialysis patients. Lower irisin levels were observed in the peritoneal dialysis group when compared to a control group, and thus the thesis was supported. ${ }^{47}$

Lower high-density lipoprotein (HDL) cholesterol levels are often linked to CKD. A correlation between lower irisin levels and decreased HDL cholesterol in individuals with CKD has also been observed. ${ }^{48,49}$

There is not much data available reflecting adropin levels in patients with CKD as a result of DM. A negative correlation of this new peptide and the progression of renal insufficiency (based on creatinine concentration, GFR and blood urea nitrogen) has been confirmed. ${ }^{50}$ Adropin may play an important anti-inflammatory function in DM patients by reducing the mRNA expression of interleukin 6 and TNF- $\alpha{ }^{50,51}$ Interestingly, a study published in 2016 focusing on adropin-associated genes in hemodialysis patients showed lower adropin concentrations in RXRA homozygotes (rs749759 and rs10776909) as opposed to an EHNO gene (rs2281997), where the adropin concentration was higher. ${ }^{52}$

\section{Conclusions}

Adropin and irisin are newly described myokines measured in human plasma in healthy and in diseased individuals. Their levels correlate with kidney function, the presence of DM, AH, and lipid status, but the exact function of adropin and irisin has not been specified yet. Further clinical and experimental studies are needed to clarify their role.

\section{References}

1. Gu K, Cowie CC, Harris MI. Diabetes and decline in heart disease mortality in US adults. JAMA. 1999;281(14):1291-1297.

2. Lewington S, Clarke R, Qizilbash N, Peto R, Collins R. Age-specific relevance of usual blood pressure to vascular mortality: A meta-analysis of individual data for one million adults in 61 prospective studies. Lancet. 2002;360(9349):1903-1913.

3. Kalaitzidis RG, Bakris GL. Prehypertension: Is it relevant for nephrologists? Kidney Int. 2010;77(3):194-200.

4. Kearney PM, Whelton M, Reynolds K, Whelton PK, He J. Worldwide prevalence of hypertension: A systematic review. J Hypertens. 2004; 22(1):11-19.

5. James PA, Oparil S, Carter BL, et al. Evidence-based guideline for the management of high blood pressure in adults: Report from the panel members appointed to the Eighth Joint National Committee (JNC 8). JAMA. 2014;311(5):507-520.

6. Harrison DG, Guzik TJ, Lob HE, et al. Inflammation, immunity, and hypertension. Hypertension. 2011;57(2):132-140.

7. Brandes RP. Endothelial dysfunction and hypertension. Hypertension. 2014;64(5):924-928.

8. Kumar KG, Trevaskis JL, Lam DD, et al. Identification of adropin as a secreted factor linking dietary macronutrient intake with energy homeostasis and lipid metabolism. Cell Metab. 2008;8(6):468-481.

9. Marczuk N, Cecerska-Heryć E, Jesionowska A, Dołęgowska B. Adropina - rola fizjologiczna i patofizjologiczna. Postepy Hig Med Dosw. 2016;70:981-988.

10. Boström P, Wu J, Jedrychowski MP, et al. A PGC1- $a$-dependent myokine that drives brown-fat-like development of white fat and thermogenesis. Nature. 2012;481(7382):463-468.

11. Huh JH, Ahn SV, Choi JH, Koh SB, Chung CH. High serum irisin level as an independent predictor of diabetes mellitus: A longitudinal population-based study. Medicine (Baltimore). 2016;95(23):e3742.

12. Desir GV, Peixoto AJ. Renalase in hypertension and kidney disease. Nephrol Dial Transplant. 2014;29(1):22-28.

13. Redwood H. Hypertension, society, and public policy. Eur Heart J Suppl. 2007;9(Suppl B):B13-B18.

14. Altincik A, Sayin O. Evaluation of the relationship between serum adropin levels and blood pressure in obese children. J Pediatr Endocrinol Metab. 2015;28(9-10):1095-1195.

15. Gu X, Li H, Zhu X, et al. Inverse correlation between plasma adropin and ET-1 levels in essential hypertension: A cross-sectional study. Medicine (Baltimore). 2015;90(40):e1712.

16. Gulen B, Eken C, Kucukdagli OT, et al. Adropin levels and target organ damage secondary to high blood pressure in the ED. Am JEmerg Med. 2016;34(11):2061-2064.

17. Fu J, Han Y, Wang J, et al. Irisin lowers blood pressure by improvement of endothelial dysfunction via AMPK-Akt-eNOS-NO pathway in the spontaneously hypertensive rat. J Am Heart Assoc. 2016;5(11).

18. Zhang LJ, Xie Q, Tang CS, Zhang AH. Expressions of irisin and urotensin II and their relationships with blood pressure in patients with preeclampsia. Clin Exp Hypertens. 2017;39(5):460-467. 
19. Zhang W, Chang L, Zhang C, et al. Central and peripheral irisin differentially regulate blood pressure. Cardiovasc Drugs Ther. 2015;29(2): 121-127.

20. Rahmouni K, Morgan DA. Hypothalamic arcuate nucleus mediates the sympathetic and arterial pressure responses to leptin. Hypertension. 2007;49:647-652.

21. American Diabetes Association. Diagnosis and classification of diabetes mellitus. Diabetes Care. 2010;33(1):62-69.

22. Choi YK, Kim MK, Bae KH, et al. Serum irisin levels in new-onset type 2 diabetes. Diabetes Res Clin Pract. 2013;100(1):96-101.

23. Shoukry A, Shalaby SM, El-Arabi Bdeer S, Mahmoud AA, Mousa MM Khalifa A. Circulating serum irisin levels in obesity and type 2 diabetes mellitus. IUBMB Life. 2016;68(7):544-556.

24. Zhang C, Ding Z, Lv G, Li J, Zhou P, Zhang J. Lower irisin level in patients with type 2 diabetes mellitus: A case-control study and meta-analysis. J Diabetes. 2016;8(1):56-62.

25. Yuksel MA, Oncul M, Tuten A, et al. Maternal serum and fetal cord blood irisin levels in gestational diabetes mellitus. Diabetes Res Clin Pract. 2014;104(1):171-175.

26. Celik E, Yilmaz E, Celik O, et al. Maternal and fetal adropin levels in gestational diabetes mellitus. J Perinat Med. 2013;41(4):375-380.

27. Beigi A, Shirzad N, Nikpour F, Nasli Esfahani E, Emamgholipour S, Bandarian F. Association between serum adropin levels and gestational diabetes mellitus: A case-control study. Gynecol Endocrinol. 2015;31(12):939-941.

28. Dąbrowski FA, Jarmużek P, Gondek A, Cudnoch-Jędrzejewska A, Bomba-Opoń D, Wielgoś M. First and third trimester serum concentrations of adropin and copeptin in gestational diabetes mellitus and normal pregnancy. Ginekol Pol. 2016;87(9):629-634

29. Aydin S, Kuloglu T, Aydin S. Copeptin, adropin and irisin concentrations in breast milk and plasma of healthy women and those with gestational diabetes mellitus. Peptides. 2013;47:66-70.

30. Liu JJ, Liu S, Wong MD, et al. Relationship between circulating irisin renal function and body composition in type 2 diabetes. $J$ Diabetes Complications. 2014;28(2):208-213.

31. Liu JJ, Wong MD, Toy WC, et al. Lower circulating irisin is associated with type 2 diabetes mellitus. J Diabetes Complications. 2013;27(4): 365-369.

32. Zhu D, Wang H, Zhang J, et al. Irisin improves endothelial function in type 2 diabetes through reducing oxidative/nitrative stresses. J Mol Cell Cardiol. 2015;87:138-147.

33. Xiang L, Xiang G, Yue L, Zhang J, Zhao L. Circulating irisin levels are positively associated with endothelium-dependent vasodilation in newly diagnosed type 2 diabetic patients without clinical angiopathy. Atherosclerosis. 2014;235(2):328-333.

34. Xiong XQ, Chen D, Sun HJ, et al. FNDC5 overexpression and irisin ameliorate glucose/lipid metabolic derangements and enhance lipolysis in obesity. Biochim Biophys Acta. 2015;1852(9):1867-1875.

35. So WY, Leung PS. Irisin ameliorates hepatic metabolism and survival in insulin-resistant human HepG2 cells through AMP-activated protein kinase signaling. Int J Biochem Cell. 2016;78:237-247.
36. Löffler D, Müller U, Scheuermann K, et al. Serum irisin levels are regulated by acute strenuous exercise. J Clin Endocrinol Metab. 2015; 100(4):1289-1299.

37. Peter PR, Park KH, Huh JY, Wedick NM, Mantzoros CS. Circulating irisin levels are not affected by coffee intake: A randomized controlled trial. PLoS One. 2014;9(4):e94463.

38. Qiu S, Cai X, Yin H, et al. Association between circulating irisin and insulin resistance in non-diabetic adults: A meta-analysis. Metabolism. 2016;65(6):825-834.

39. Aydin S, Kuloglu T, Aydin S, et al. Expression of adropin in rat brain, cerebellum, kidneys, heart, liver, and pancreas in streptozotocininduced diabetes. Mol Cell Biochem. 2013;380(1-2):73-81.

40. Topuz M, Celik A, Aslantas T, Demir AK, Aydin S, Aydin S. Plasma adropin levels predict endothelial dysfunction like flow-mediated dilatation in patients with type 2 diabetes mellitus. J Investig Med. 2013; 61(8):1161-1164.

41. Wu L, Fang J, Chen L, et al. Low serum adropin is associated with coronary atherosclerosis in type 2 diabetic and non-diabetic patients. Clin Chem Lab Med. 2014;52(5):751-758.

42. Levey AS, Atkins R, Coresh J, et al. Chronic kidney disease as a global public health problem: Approaches and initiatives. A position statement from Kidney Disease Improving Global Outcomes. Kidney Int. 2007;72(3):247-259.

43. Levey AS, Coresh J. Chronic kidney disease. Lancet. 2012;379(9811): 165-180.

44. Yang S, Xiao F, Pan L, et al. Association of serum irisin and body composition with chronic kidney disease in obese Chinese adults: A crosssectional study. BMC Nephrol. 2015;16:16.

45. Rodríguez-Carmona A, Pérez Fontán M, Sangiao Alvarellos $S$, et al. Serum levels of the adipomyokine irisin in patients with chronic kidney disease. Nefrologia. 2016;36(5):496-502.

46. Moraes C, Leal VO, Marinho SM, et al. Resistance exercise training does not affect plasma irisin levels of hemodialysis patients. Horm Metab Res. 2013;45(12):900-904.

47. Lee MJ, Lee SA, Nam BY, et al. Irisin, a novel myokine, is an independent predictor for sarcopenia and carotid atherosclerosis in dialysis patients. Atherosclerosis. 2015;242(2):476-482.

48. Holzer M, Birner-Gruenberger R, Stojakovic T, et al. Uremia alters HDL composition and function. J Am Soc Nephrol. 2011;22(9):1631-1641.

49. Wen MS, Wang CY, Lin SL, Hung KC. Decrease in irisin in patients with chronic kidney disease. PLoS One. 2013;8(5):e64025.

50. Hu W, Chen L. Association of serum adropin concentrations with diabetic nephropathy. Mediators Inflamm. 2016;2016:6038261.

51. Akcilar R, Kocak FE, Simsek H, et al. Antidiabetic and hypolipidemic effects of adropinin streoptozotocin-induced type 2 diabetic rats. Bratis/ Lek Listy. 2016;117(2):100-105.

52. Grzegorzewska A, Niepolski L, Mostowska A, WarchołW, Jagodziński P. Involvement of adropin and adropin-associated genes in metabolic abnormalities of hemodialysis patients. Life Sci. 2016;160:41-46. 